\title{
A physical background model for the Fermi Gamma-ray Burst Monitor
}

\author{
B. Biltzinger, F. Kunzweiler ${ }^{\star}$ J. Greiner, K. Toelge ${ }^{\star \star}$, and J. Michael Burgess
}

Max-Planck-Institut für Extraterrestrische Physik, Giessenbachstrasse 1, 85748 Garching, Germany
e-mail: bbiltzing@mpe.mpg.de

Received 18 December 2019 / Accepted 20 May 2020

\begin{abstract}
We present the first physically motivated background model for the Gamma-ray Burst Monitor (GBM) on board the Fermi satellite. Such a physically motivated background model has the potential to significantly improve the scientific output of Fermi/GBM, as it can be used to improve the background estimate for spectral analysis and localization of gamma-ray bursts (GRBs) and other sources. Additionally, this model can also lead to detections of new transient events, since long and weak, or slowly rising, events do not activate one of the existing trigger algorithms. In this paper we show the derivation of such a physically motivated background model, which includes the modeling of the different background sources and the correct handling of the response of GBM. While the goal of the paper is to introduce the model rather than developing a transient search algorithm, we demonstrate the ability of the model to fit the background seen by GBM by showing the following four applications for (1) a canonical GRB, (2) the ultra-long GRB 091024, (3) the V404 Cygni outburst in June 2015, and (4) the ultra-long GRB 130925A.
\end{abstract}

Key words. instrumentation: detectors - methods: data analysis - methods: statistical - gamma rays: general

\section{Introduction}

The background seen by astrophysical instruments can be very hard to model, as it normally contains a plethora of unmodeled sources, instrumental background, and general unknowns. Nevertheless, these background signals can contain information which, when modeled, can be extracted for scientific study. For instruments with the ability to spatially separate source and background via, for example, focusing techniques, the characterization of background signals can be simple, at least in the sense that spatially distinct sources of background emission can readily be identified. When an instrument lacks imaging capabilities in the classical sense, that is, its data are all-sky light curves with sources superimposed upon each other, modeling and separation of background sources must rely on temporal information such as the location of the instrument or viewing direction with time. Then sources of background emission can be modeled and pushed through this temporal response of the instrument.

The Gamma-ray Burst Monitor (GBM) is part of the described class of non-imaging instruments. The GBM is one of two instruments that are part of the Fermi Gamma-ray Space Telescope, which was launched in 2008 into a low-Earth orbit. This instrument consists of 12 sodium iodide (NaI) and 2 bismuth germanate (BGO) detectors, pointing in different directions (see Fig. 1). The GBM has an all-sky coverage, except for the part that is occulted by the Earth. The detectors nominally cover an energy interval from $8 \mathrm{keV}$ to $1 \mathrm{MeV}$ for the $\mathrm{NaI}$ detectors and $150 \mathrm{keV}-40 \mathrm{MeV}$ for the BGO detectors (Meegan et al.

^ Present address: Technische Universität München, Arcisstraße 21, 80333 München, Germany.

$\star \star$ Present address: Capgemini Nederland B.V., Reykjavikplein 1, 3543 KA Utrecht, The Netherlands.
2009). The GBM is used to detect and spectrally analyze transient events. It provides an extension of the energy range over which transient events can be observed below the Large Area Telescope (LAT) energy range, which covers $20 \mathrm{MeV}-300 \mathrm{GeV}$; also it allows fast onboard localization of transients to point the LAT toward the transient event to detect potential high-energy delayed emission (Meegan et al. 2009). As a non-imaging instrument, GBM is inundated with an all-sky background composed of several constant and temporally evolving sources. Thus, for the identification of temporal transients such as gamma-ray bursts (GRBs), GBM relies on the ability to separate source and background in any analysis. The classical approach in estimating the background temporally and spectrally is to identify off-source regions in time and fit smooth functions such as polynomials to temporally evolving signal of each pulse height analysis (PHA) channel. Then, the estimated model is extrapolated into the on-source region, thus providing an estimate for the background in that time interval (Pendleton et al. 1999; Greiner et al. 2016).

This classical approach is suitable for transient events that are short with respect to the typical temporal variation of the background (about $10 \mathrm{~min}$ ), but can be very problematic for long duration events, as the background extrapolation can be poor and inaccurate due to non-polynomial variations in the signal. Moreover, assuming independent temporal variations for each channel allows for a high degree of freedom that is unlikely to exist. An alternative approach is to construct a background model from physical components that evolve temporally and spatially. Such a model can be complex because of the many different background components; this complexity explains why after 12 years of operation of GBM and 28 years after the CGRO/BATSE observations, there is still no properly working physical model to describe the background of GBM. 
Some empirical attempts have been made in the past: (1) Szécsi et al. (2013) fit the background around trigger times of GBM with separate polynomials (up to third order) to different quantities such as the angle between the detector and the Earth, forming a geometrical background model. But this approach gives the fit a lot of freedom because it has many free unbound parameters, introduces ambiguity between the different polynomials, and does not use physical information of the sources that create the background. Another work that used the background seen by GBM was done by (2) Horiuchi et al. (2015). In this work the authors searched for decay lines of sterile neutrinos in the background spectrum of GBM. They used data cuts to get rid of most of the background contamination from, for example, cosmic rays and the Earth albedo, but did not try to model all the background sources. (3) Fitzpatrick et al. (2012) used the fact that the detectors of GBM, in most cases, are at the same geographical coordinates and point to the same position in the sky every 30th orbit. Thus, these authors assume that the background at time $T$ should be the same as at time $T \pm 30$ orbits. This is a purely empirical method that for example breaks down, as stated in Fitzpatrick et al. (2012), when there is an autonomous repoint request (ARR) or when the particle flux changes or the Earth's magnetic field is compressed owing to varying solar activity. An ARR causes the Fermi satellite to slew in order to point the LAT toward a GRB. If this happens, the pointing direction of the GBM detectors will not be the same 30 orbits later.

We wanted to build a predictive background model with a minimum number of free parameters, which incorporates the physics of the source types and the response of the GBM detectors. Such a model should only fit the background and not (long or slowly varying) transient signals, and should allow us to determine the individual contributions of the different background sources. The model presented herein treats all its components physically except the cosmic-ray background, which is explained in Sect. 3.3.5. One possible future application for a physically motivated background model could be the possibility to detect events with slow raising count rates (e.g., ultralong GRBs). Herein, we show some examples of the background seen by GBM in Sect. 2, describe the setup up of such a physically motivated model in Sect. 3, and show results for the ultra-long GRB 091024, the 2015 V404 Cygni outburst, and GRB 110920A in Sect. 4.

\section{GBM and its background}

The GBM converts detected photon events into 128 PHA channels according to the "height" of the electronic signal at the end of the photomultiplier tube. These signals are then stored as single events with time tags and the corresponding PHA channel number in the Time-Tagged Events (TTE) data files, but also binned in $8 \mathrm{~s}$ time bins with full spectral resolution of $128 \mathrm{PHA}$ channels (so-called CSPEC data files) and with $256 \mathrm{~ms}$ time bins with a reduced spectral resolution of only 8 PHA channels (socalled CTIME data files). The continuous TTE data only exist since 2010 (Meegan et al. 2009).

The background seen by the individual detectors of GBM (see Fig. 1) is a superposition of several different background sources, whose contributions to the total background are strongly dependent on the energy and orientation of the detectors with respect to the position of the background sources; more details about the different background sources are described in Sect. 3.3. Since the off-axis sensitivity is energy-dependent, the same photon spectrum results in different count spectra depending on the orientation of the detector with respect to the source posi-

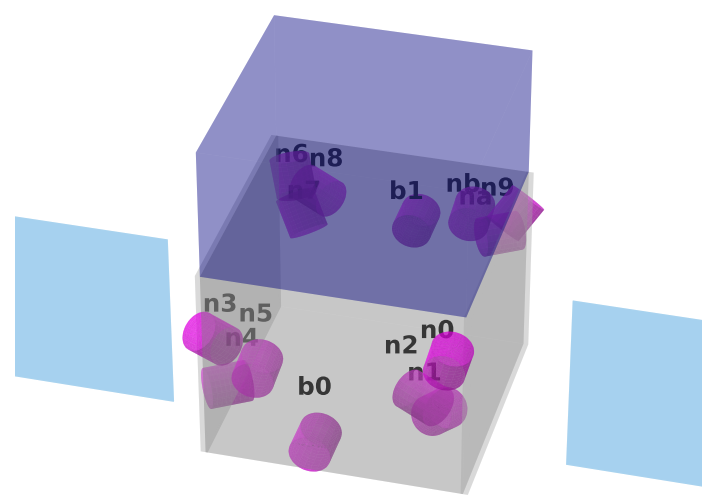

Fig. 1. Schematic picture of the setup of GBM on board the Fermi satellite. The mounting points and pointing directions for all 14 detectors that are part of GBM are shown. Additionally the satellite base is indicated in gray, the LAT is shown in dark blue, and the solar panels are indicated in light blue.

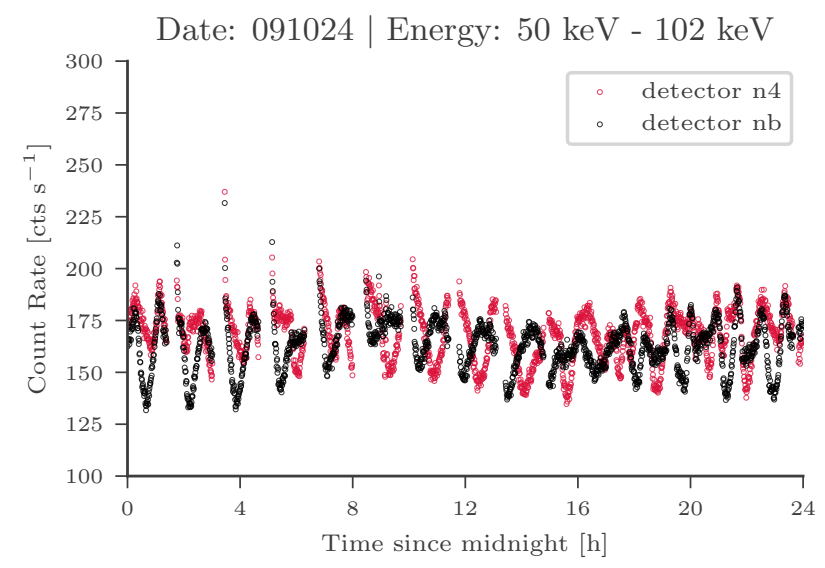

Fig. 2. Background variation at a binning of $15 \mathrm{~s}$ for the date 24 October 2009 for the two detectors $\mathrm{n} 4$ (red circles) and nb (black circles) covering the same reconstructed energy range $50 \mathrm{keV}-102 \mathrm{keV}$.

tion. The dependence on the orientation leads to different background variations for different detectors in the same reconstructed energy range, while the dependence on the energy leads to different background variations for the same detector but different reconstructed energy ranges. This is shown in Figs. 2 and 3. The background model needs to be capable of explaining these different backgrounds for different detector orientations and PHA channels.

\section{Model}

\subsection{Response}

For spectral instruments with large energy dispersion such as GBM, modeling the response is crucial for any physical interpretation of the data. The response gives the effective area seen by a photon with a given energy $E_{\mathrm{ph}}$ to be detected in one of the detector's PHA channels $E_{\mathrm{det}}$, and therefore connects the physical spectrum of the source with the count rates in the PHA channels. The effects that are incorporated in the response include, on the single detector level, the geometry of the scintillation crystal, energy dispersion, partial energy deposition of the photons in the crystal, and absorption of photons by the detector housing and photomultiplier tubes attached to the crystal. Additionally, on 


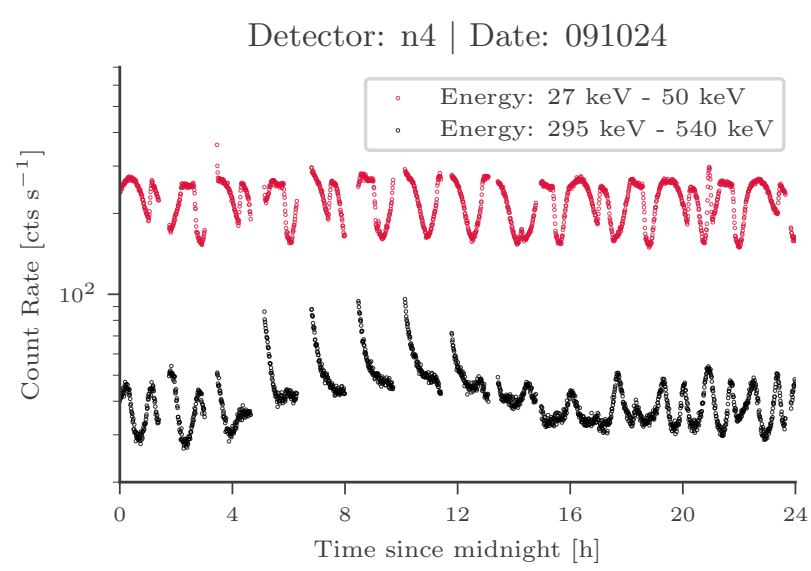

Fig. 3. Background variation at a binning of $15 \mathrm{~s}$ for the date 24 October 2009 for the detector $\mathrm{n} 4$ and for the two reconstructed energy ranges $27 \mathrm{keV}-50 \mathrm{keV}$ (red circles) and $295 \mathrm{keV}-540 \mathrm{keV}$ (black circles).

the satellite level, shielding by other components of the satellite and other detectors is taken into account (see Fig. 4, where for example the influence of the LAT is clearly visible in the upper left plot, as it cuts away the effective area on one side of the line of sight.). The response is therefore a function of the position of a source in the satellite frame, the energy of the incoming photon, and the PHA channel in which the photon is detected (Kippen \& Hoover 2007).

Each PHA channel has an associated reconstructed energy range, but this does not imply that all photons detected in this PHA channel have a physical energy within the reconstructed energy range associated with this PHA channel owing to energy dispersion and partial energy deposition of the photon in the crystal.

The connection of the physical spectrum $F$ [photons $\mathrm{cm}^{-2} \mathrm{~s}^{-1}$ ] and the detected count rate spectrum $D$ [counts $\mathrm{s}^{-1}$ ] via the response $R$ is given in Eq. (1),

$D\left(E_{\mathrm{det}}\right)=\int \mathrm{d} E_{\mathrm{ph}} R\left(E_{\mathrm{ph}}, E_{\mathrm{det}}, \phi_{\text {source }}, \theta_{\text {source }}\right) \cdot F\left(E_{\mathrm{ph}}\right)$.

The quantities $\phi_{\text {source }}$ and $\theta_{\text {source }}$ define the position of the source in the satellite frame, where $\theta_{\text {source }}$ is the zenith angle and $+\phi_{\text {source }}$ the azimuth angle measured from the pointing direction of the b0 detector.

To determine the detector response matrix (DRM), an onground calibration of GBM was performed (Bissaldi et al. 2009). We used the Python package gbm_drm_gen ${ }^{1}$ to get the DRMs. The package gbm_drm_gen uses the responses generated via simulation to match the calibration of GBM. With its help we can calculate the DRMs for user defined energy bins of the incoming photons, detector PHA channels, and positions of the source (see Fig. 5) (Burgess et al. 2018; Burgess 2019; Berlato et al. 2019).

\subsection{Response implementation}

In the physical background model the temporally changing responses for point sources (e.g., the Sun) and for extended sources (e.g., cosmic gamma-ray background) are calculated to get the correct influence of these sources on the count rates. The implementation of the response for a point sources is, with the help of the gbm_drm_gen package, straightforward:

\footnotetext{
1 https://github.com/grburgess/gbm_drm_gen/
}

1. Calculate the position of the point source in the satellite frame several times with an appropriate time resolution (computation time versus accuracy of interpolation in step 4).

2. Use the gbm_drm_gen package to get the DRMs for the different positions.

3. Convolve the assumed spectrum with the DRMs to get the expected count rates in the different PHA channels.

4. Interpolate between the times for which the point source position was calculated.

The use of an interpolation in time in the last step will of course introduce a systematic error in our analysis. In our implementation, 800 time steps are used for one day, which results in one time step every $108 \mathrm{~s}$. In this short time the direction of the point source moves only very little in the satellite coordinate frame. Therefore the response change between two time steps is very small, which is why the introduced systematic error is small. There are some regions of the GBM response with fast changes for similar directions of the incident photon, which are caused by some parts of the satellite such as the LAT radiators. This may introduce a larger error at these times, but we checked that the results do not change if we make the time steps smaller. For the extended sources this procedure has to be modified because the response is a function of the position of the source in the satellite frame and extended sources cover a range of positions. The formula to connect the flux $F$ [photons $\mathrm{cm}^{-2} \mathrm{~s}^{-1} \mathrm{sr}^{-1}$ ] of the extend sources and the detected count rate spectrum $D$ [counts $\mathrm{s}^{-1}$ ] is given by

$D\left(E_{\mathrm{det}}\right)=\iint \mathrm{d} E_{\mathrm{ph}} \mathrm{d} \Omega R\left(E_{\mathrm{ph}}, E_{\mathrm{det}}, \phi, \theta\right) \cdot F\left(E_{\mathrm{ph}}\right) \cdot f(\phi, \theta)$,

where $f(\phi, \theta)$ is the occultation function

$f(\phi, \theta)= \begin{cases}1, & \text { if the point is occulted by the source } \\ 0, & \text { otherwise. }\end{cases}$

For a point source $f(\phi, \theta)=\delta\left(\phi-\phi_{\text {source }}\right) \delta\left(\theta-\theta_{\text {source }}\right)$, with which Eq. (2) simplifies to Eq. (1).

To get the expected count rate spectrum of an extended source, the following procedure is applied:

1. Build a grid with $N_{\text {grid }}$ equally distributed points on a unit sphere around the detector in the satellite frame.

2. For each of these points, calculate the DRM with the gbm_drm_gen package.

3. Calculate the region covered by the source in the satellite frame several times with appropriate time resolution (balancing computation time with accuracy of the interpolation in step 6).

4. Sum the responses of the relevant grid points and multiply the result by the solid angle that every point covers $\left(\frac{4 \pi}{N_{\text {grid }}} \mathrm{sr}\right)$ to get an effective response.

5. Convolve the assumed spectrum through the effective response to get the expected count rates in the different PHA channels.

6. Interpolate between the times for which the count rate was calculated.

With the response effects for point sources and extended sources implemented, we can predict the count rate spectrum for any given physical spectrum of any source.

The intense flux of photons from a GRB scatters of the Earth's atmosphere and produce a secondary, weaker flux of photons that can be detected by GBM. Indeed all high-energy radiation can produce such a secondary flux, but at levels far below 

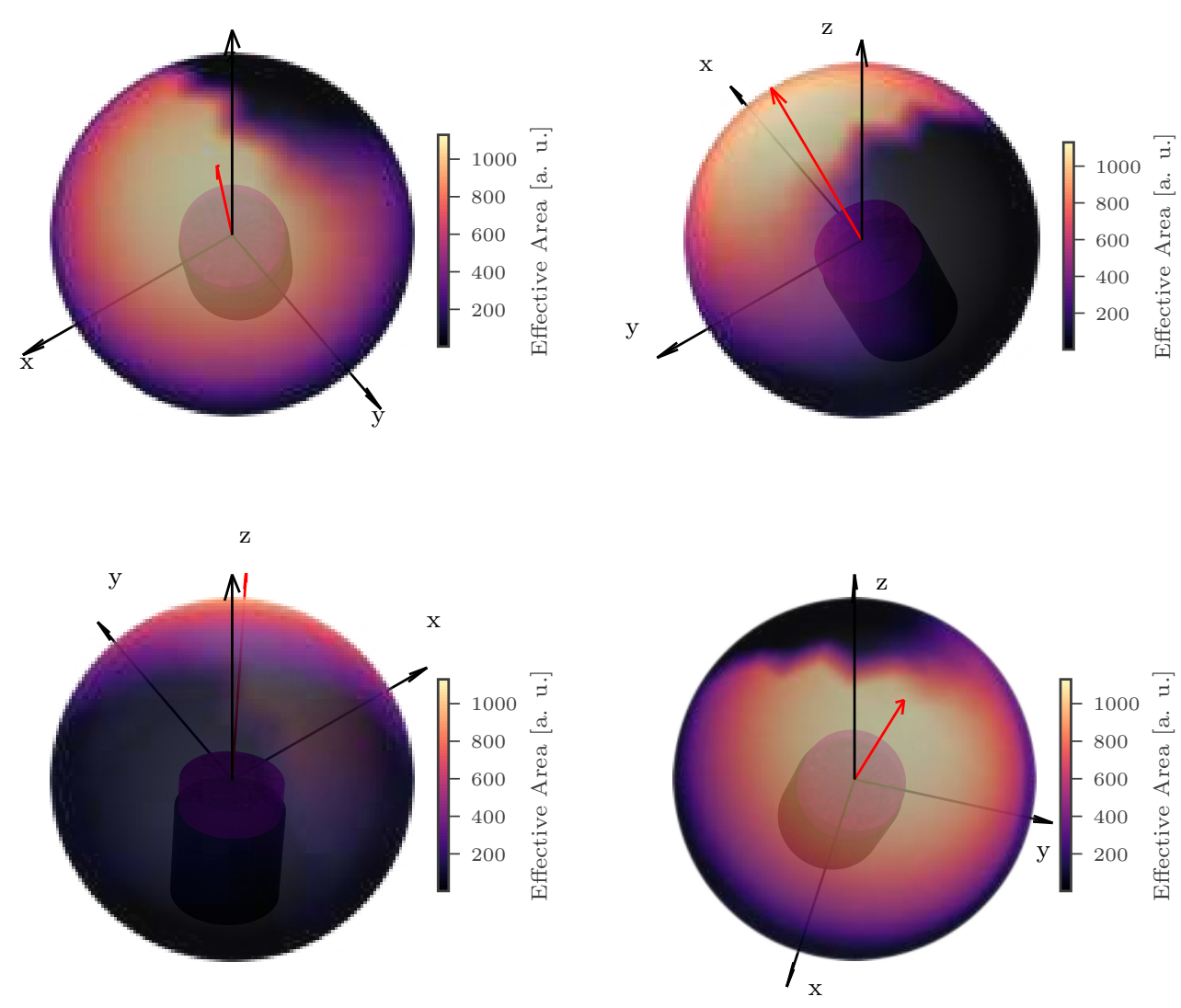

Fig. 4. Effective area seen by a photon with energy $38 \mathrm{keV}$ to be detected in the reconstructed energy channel 2 (CTIME) for different arrival directions on a unit sphere around detector $\mathrm{n} 0$ for four different viewing directions on the sphere. The red arrow marks the line of sight of the detector n0 (optical axis), the dark cylinder symbolizes the detector housing and the magenta cylinder the detector crystal. The coordinate system is defined such that the $z$-axis is the pointing direction of the LAT and the $x$-axis points along the normal of the satellite side on which the b0 detector is mounted. Responses generated with gbm_drm_gen (Burgess et al. 2018; Burgess 2019; Berlato et al. 2019).

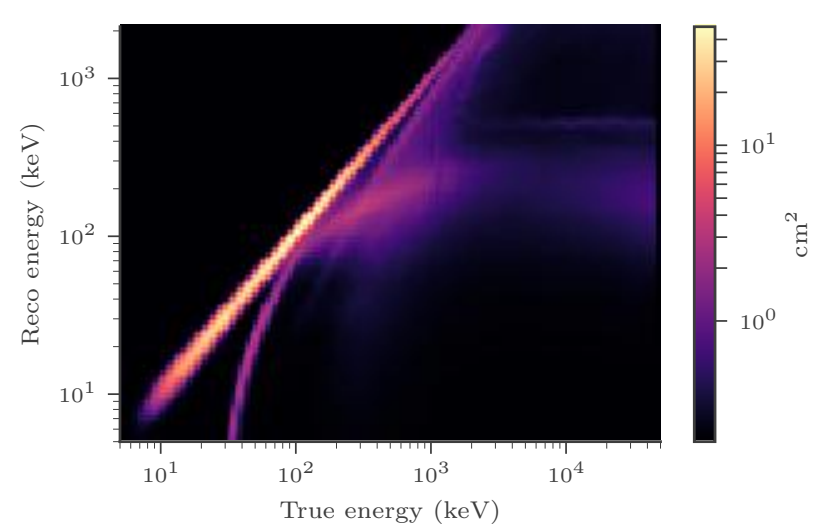

Fig. 5. Example response that shows the effective area for different photon energies to be detected in the PHA channels with associated reconstructed energy. Figure created with the gbm_drm_gen package (Hoover et al. 2005; Burgess 2019).

that of a GRB; therefore, we neglect atmospheric scattering from non-GRB sources in this work.

\subsection{Background sources}

Our model incorporates six components which are described in this subsection. We start with the simplest model of a constant background and then add more components one at a time. For each step we show a fit of the model with the introduced background components to the data of detector n6 for 26 October 2009 between 102 and $295 \mathrm{keV}$ reconstructed energy, showing the improvement we can get by adding the different components to the model. The used components can be categorized into two main classes: the photon and the charged particle background components. The first class is characterized by the components producing a photon spectrum, which is directly measured by the GBM detectors. Members of this class are the Earth albedo, the cosmic gamma-ray background (CGB), point sources, and the Sun. In the second class, containing cosmic rays and the South Atlantic Anomaly (SAA), charged particles alter the background owing to excitation of satellite atoms or via direct energy deposit in the detectors.

\subsubsection{Constant background}

The most intuitive model is that of a constant background, which is shown in Fig. 6, plus gaps during every SAA transition when the detectors are shut down.

When building a physically motivated background model for GBM, for instance, to detect new sources, it is reasonable to start by fitting for the sharply peaked and rapidly decaying signal that occurs after the exits of the SAA. This is our first extension to the constant background model.

\subsubsection{South Atlantic Anomaly}

There are two regions in the Earth's magnetic field that have the right properties to permanently trap large densities of charged particles (mostly protons and electrons) in radiation belts. These two regions are called inner and outer Van Allen belts and both lie, outside of the SAA, well above Fermi's orbit, which has an altitude of about $550 \mathrm{~km}$; the inner Van Allen belt is located between $1000 \mathrm{~km}$ and $6000 \mathrm{~km}$ (van Allen \& Frank 1959; da Silva \& Rocco 2017; Ganushkina et al. 2011).

The SAA is a region located over the South Atlantic, where the Earth's magnetic field is significantly weaker (Schaefer et al. 2016). This originates from an offset of the dipole, which 


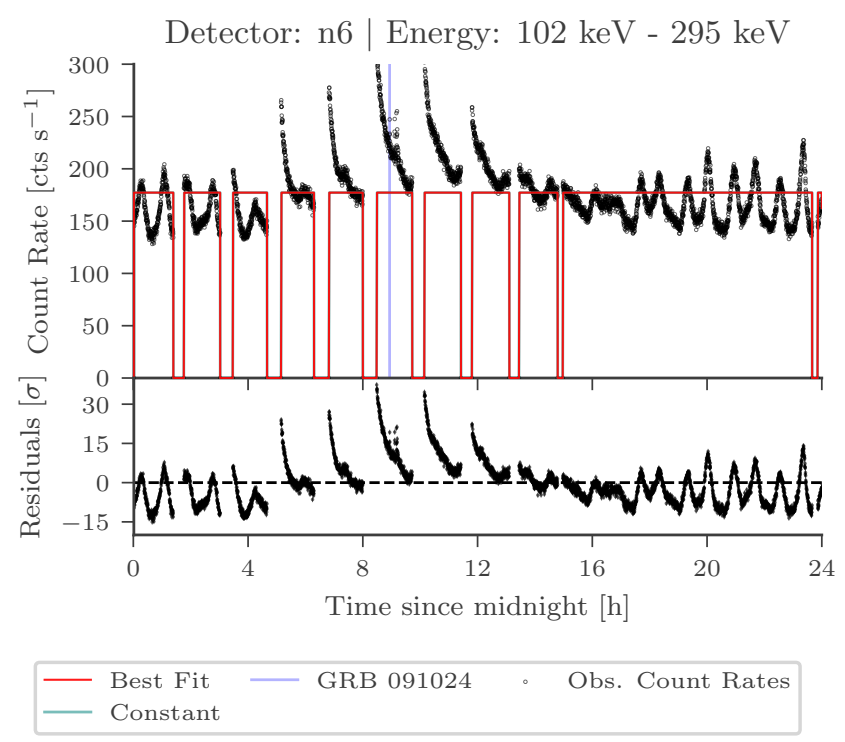

Fig. 6. Fit of a constant line to the data of detector $\mathrm{n} 4$ and the energy of $295 \mathrm{keV}-540 \mathrm{keV}$ of day 26 January 2015 . The count rate of the model has been set to zero during the SAA passage where the detectors are turned off. The GBM trigger time for GRB 091024 is denoted by a cyan line.

approximates Earth's magnetic field and causes the inner Van Allen belt to bend toward the Earth at the position of the SAA (Mozzoni 2007); the Van Allen belt reaches an altitude as low as $100 \mathrm{~km}$ and therefore intersects Fermi's orbit at $550 \mathrm{~km}$. In order to protect the detectors from damage caused by the high flux of charged particles, the detectors are routinely shut down when the satellite crosses the SAA. Nevertheless, the satellite and detector material undergo nuclear excitation by collision with the charged particles and subsequently photons are produced by the de-excitation of the activated material, which is measured when the detectors are turned on again. As the high count rates after the SAA originate from nuclear de-excitation, its influence should decay exponentially over time and should have the functional form

$R_{\mathrm{SAA}}(t)=R_{\mathrm{SAA}}\left(t_{0}\right) \cdot \exp \left(-C_{\text {decay }} \cdot\left(t-t_{0}\right)\right)$

Because of the different elements in the material found in the satellite and the detector, there should be a superposition of several exponential decays after each SAA exit. To keep the computational effort manageable, we included only two decay functions after each exit to allow for a fast and a slow decay. The fast decaying part usually decays in $\approx$ minutes, and is thus completely gone before the next SAA passage. But the slowly decaying part can take several hours to decay completely, which causes accumulation of background signal by consecutive SAA passages. This activation decays slowly during the long time of the day with no SAA passage. This leads to four parameters per SAA exit: one for each decay constant and the two corresponding normalizations. It is clearly visible in Fig. 7 that the model is able to explain the rapidly decaying count rates after the SAA passage, and is significantly reducing the peaks in the residuals.

The presented procedure for the SAA influence depends on the boundaries, which are used by the Fermi team, being correct. If the boundaries of the SAA are wrong, for example, the detectors can be turned off a bit too late, which leads to a rise in the count rates before the official SAA entrance.

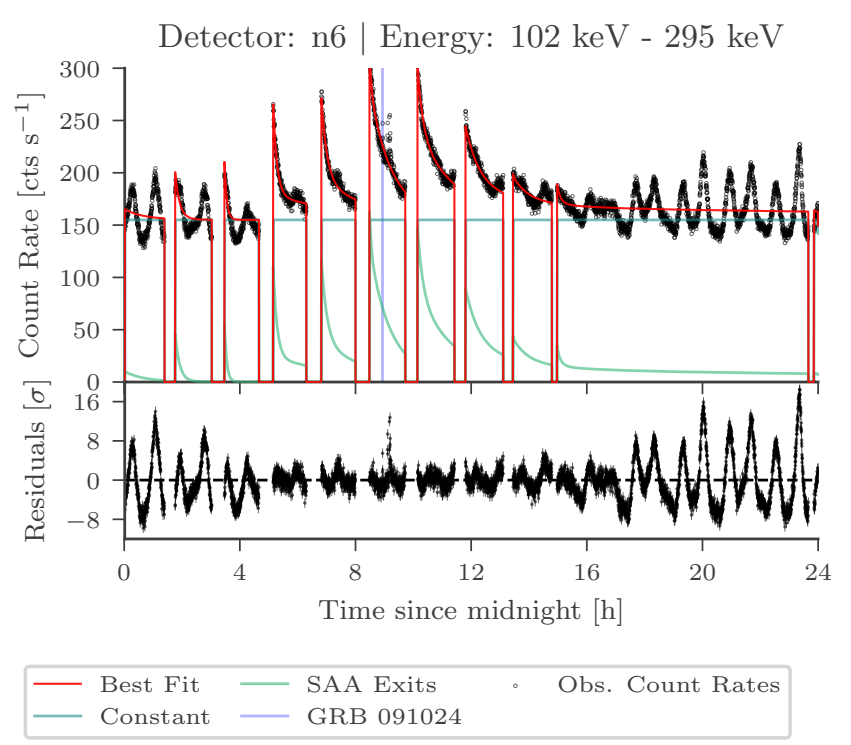

Fig. 7. Same as Fig. 6, but including the additional SAA component. The GBM trigger time for GRB 091024 is denoted by a cyan line.

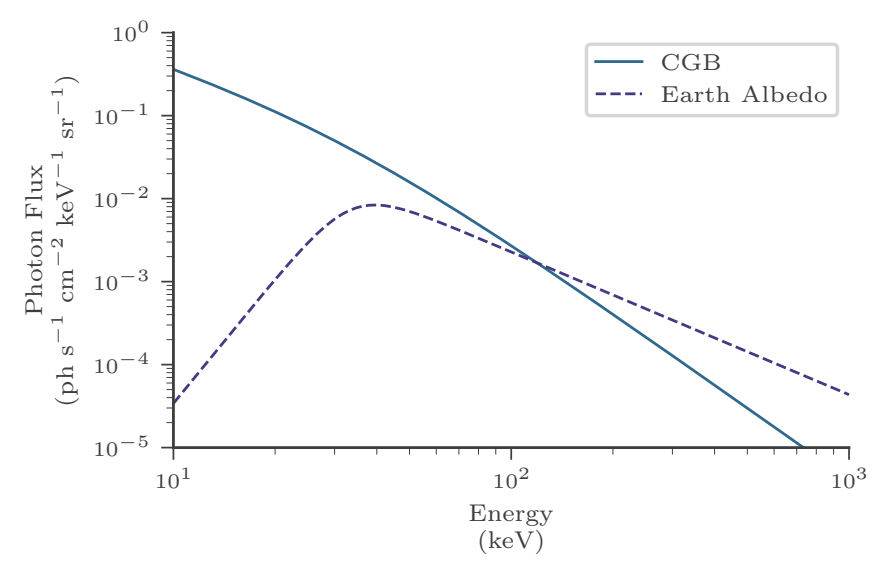

Fig. 8. Spectra of the CGB and the Earth albedo for spectral parameters given in Ajello et al. (2008).

As the SAA shape and dimension changes owing to solar activity and is also slowly moving over the years, the Fermi team is adapting the SAA boundaries as needed, so that such outliers should be very small in number. To be on the safe side we could always ignore some time (few hundreds of seconds) before and after the official SAA transition.

\subsubsection{Earth albedo}

The Earth is known to be a source of gamma-rays in the GBM energy range (Thompson et al. 1981; Ajello et al. 2008). These gamma-rays are produced by cosmic rays colliding with molecules in the atmosphere of the Earth through $\pi^{0}$-decay and bremsstrahlung (Petry 2005; Abdo et al. 2009). The spectrum of the produced gamma-rays that are emitted back into space is called the Earth albedo and is shown in Fig. 8 in comparison to the cosmic gamma-ray spectrum. The spectrum of the CGB is higher than that of the Earth albedo for energies smaller than $\approx 120 \mathrm{keV}$, but for higher energies the spectrum of the CGB has a steeper slope, which causes the Earth albedo to surpass the CGB spectrum for energies higher than $\approx 120 \mathrm{keV}$. 


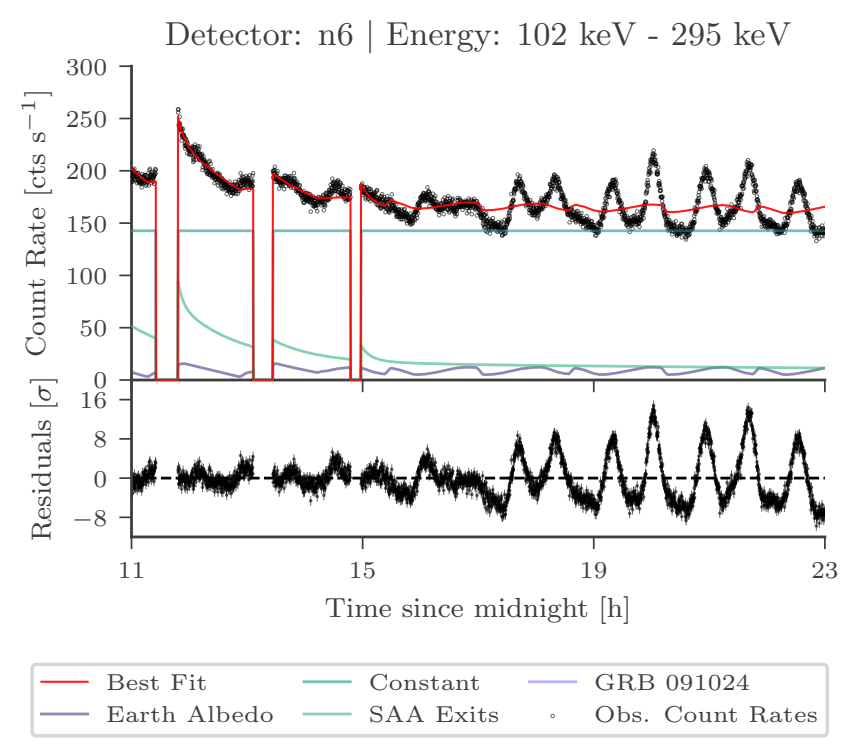

Fig. 9. Same as Fig. 7 but only for half of the day for better legibility and including the Earth albedo as an additional background source.

The secondary particles produced in the collision of a cosmic ray with an atmospheric molecule tend to fly in the same direction as the primary cosmic ray because of momentum conservation. As cosmic rays mostly consist of protons, they get deflected eastward upon impinging Earth's magnetic field, therefore the cosmic-ray flux from the west as seen by the satellite is larger. It has been shown by Petry (2005) that the east-west asymmetry in the Earth albedo peaks at a photon energy in the GeV range and is small at lower energies in the MeV range. Abdo et al. (2009) showed that the effect is also very small for very high energies $(\approx \mathrm{TeV})$. Additionally, nearly the whole photon flux with very high energies $(\approx \mathrm{TeV})$ originates from a small ring around the Earth (as seen by the satellite). This "ring effect" weakens toward lower gamma-ray energies; therefore we assume that this effect can be neglected in the $\mathrm{keV}$ to low $\mathrm{MeV}$ region (Abdo et al. 2009).

It is therefore reasonable to assume that the east-west and ring effects, which are well observed at higher energies, are not significant in the $\mathrm{keV}$ to low $\mathrm{MeV}$ energy region and therefore to assume that the Earth albedo at these energies is isotropic over the whole Earth surface seen by the satellite.

The variation of the background from the Earth albedo in the individual detectors is caused by the different positions of the Earth in the satellite coordinate system for different times and the therefore changing effective response (see Sect. 3.2). Fig. 9 shows the model expanded by the component for the Earth albedo. The assumed functional form of the Earth albedo spectrum in this paper is a smoothly connected double power law with the spectral parameter values as given in Ajello et al. (2008), but we free the normalization $C$ and fit it to the GBM data as follows:

$\frac{\mathrm{d} N}{\mathrm{~d} E}=\frac{C}{\left(\frac{E}{33.7 \mathrm{keV}}\right)^{-5}+\left(\frac{E}{33.7 \mathrm{keV}}\right)^{1.72}}$.

\subsubsection{Cosmic gamma-ray background}

The CGB, which was discovered more than 50 years ago, is generally agreed to be produced by emission of unresolved

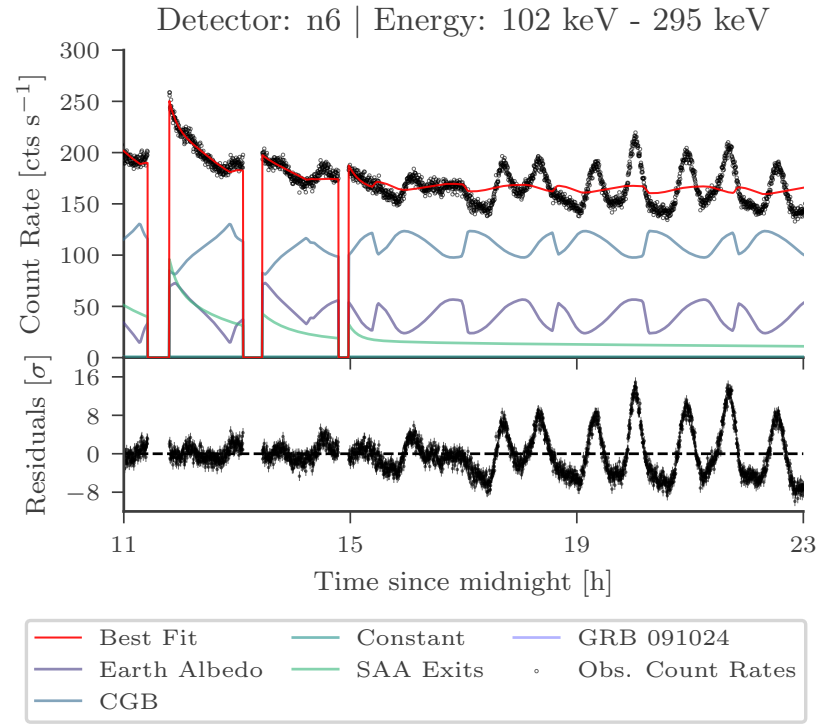

Fig. 10. Same as Fig. 9 but including the CGB as additional background source.

extragalactic point sources (Giacconi et al. 1962; Hasinger 2004; Ajello et al. 2008). The galactic contribution to the CGB is subdominant for photon energies below $1 \mathrm{MeV}$ (Bouchet et al. 2011; Ajello et al. 2008) and almost the entire cosmic X-ray background (CXB) for energies smaller than $2 \mathrm{keV}$ is accounted for by active galactic nuclei (AGN) that host accreting supermassive black holes (Hasinger 2004). For energies above $6 \mathrm{keV}$, on the other hand, the fraction of CGB emission that can be resolved into AGN is smaller than 50\% (Worsley et al. 2005). This unresolved CGB flux could be explained by photon emission of a yet undetected population of highly absorbed AGN. The spectrum of the CGB is shown in Fig. 8 for the spectral parameters given in Ajello et al. (2008).

Fig. 10 shows the model expanded by the component for the CGB. The assumed functional form of the CGB spectrum in this paper is a smoothly connected double power law with the spectral parameter values as given in Ajello et al. (2008), but we free the normalization $C$ and fit it to the data (see Eq. (6)).

$\frac{\mathrm{d} N}{\mathrm{~d} E}=\frac{C}{\left(\frac{E}{30 \mathrm{keV}}\right)^{1.32}+\left(\frac{E}{30 \mathrm{keV}}\right)^{2.88}}$.

The influence of the Earth albedo and the CGB are strongly correlated. When the influence of the earth albedo is high, the Earth is covering a part of the response with high effective area and blocking the CGB photons from this direction, and therefore leads to a lower influence of the CGB.

In addition, Churazov et al. (2008) showed, using a theoretical approach, that a significant percentage of the CGB spectrum in the $\mathrm{keV}$ range gets reflected by the Earth. As this is a second order influence it is currently not included in the work, but we plan to include this reflection of the CGB from the Earth in the future.

\subsubsection{Cosmic rays}

Cosmic rays that hit the satellite can either leave a direct signal in the detectors that is converted into a certain PHA channel or excite atoms in the satellite material that upon de-excitation produce photons (as shown for INTEGRAL/SPI in Diehl et al. 2018), which can be measured by the GBM detectors. Because 
of Fermi's low-Earth orbit and the small inclination, the detectors are protected well by the Earth's magnetic field against cosmic rays. Only the cosmic rays with very high energies $(>\mathrm{GeV}$ for protons) can reach the satellite (Pesce-Rollins 2009).

This shielding of cosmic rays varies during the orbital motion with the different magnetic field strength of the Earth in different orbital positions and therefore the amount and the spectrum of cosmic rays hitting the satellite is time dependent. We introduce two different approaches to model the cosmic-ray contribution: the McIlwain $L$-parameter and the data in the highenergy BGO channels.

The McIlwain L-parameter (McIlwain et al. 1966) can be used as quantifier to model this shielding effect against cosmic rays by the Earth's magnetic field as has been shown for example for protons and electrons measured by the LAT in Pesce-Rollins (2009). The McIlwain $L$-parameter is connected to the magnetic cutoff rigidity $R_{C}$ as given in Eq. (7). The parameters $K$ and $\alpha$ change with time and have to be determined for the corresponding time (Shea et al. 1987),

$R_{C}=K L^{-\alpha}$.

If we extend the magnetic field line at a position to the magnetic equator, then the $L$-parameter at that position gives the distance of the magnetic field line at the magnetic equator to the Earth center in units of Earth radii. The correct functional dependence of the background rate caused by cosmic rays on the $L$-parameter is not known. All that is known a priori is that a higher $L$-parameter means a weaker protection by the Earth magnetic field and thus should lead to a higher background caused by cosmic rays. We found that assuming a linear relationship between the background rate difference and the $L$-parameter difference seems to describe the detected background rates very well for the GBM detectors and the $L$-parameter range on the Fermi orbit $(\approx 1-1.7$ outside of the SAA, as shown in Pesce-Rollins 2009), that is,

$R\left(L_{1}\right)-R\left(L_{2}\right) \propto L_{1}-L_{2}$.

For cosmic rays it is assumed that Eq. (8) describes the difference in the count rates for different $L$-parameter values. We used the weekly spacecraft file ${ }^{2}$ to get the McIlwain $L$-parameter values for the different times. Parameter values are provided with a time resolution of $30 \mathrm{~s}$. Between these time steps a linear interpolation is used. Because we only have a formula for the difference in the rate for different $L$-parameters, the total rate due to the cosmic rays is defined as

$R_{\mathrm{CR}}=R_{\mathrm{CR}}\left(L_{\text {min }}\right)+C_{\text {Mcllwain }} \cdot\left(L-L_{\text {min }}\right)$,

where $L_{\min }$ is the minimal $L$-parameter of the data. Therefore, the background model component has two parameters: Firstly, a constant $R_{\mathrm{CR}}\left(L_{\mathrm{min}}\right)$, which is the same for all time bins, and secondly, a normalization $C_{\text {McIlwain, }}$ which is multiplied by the difference of the $L$-parameter of the time bin and the minimal $L$-parameter in the data. This constant contribution motivates the use of our constant source we introduced in Sect. 3.3.1. The influence of the cosmic rays in our model is direction independent. This seems to be true to first order, but we see in the highenergy channels, where the cosmic-ray contribution is dominant, that directional differences of a few tens of percent that cannot be explained by our model. This could be explained by the eastwest asymmetry of the cosmic-ray flux owing to the magnetic field of the Earth (see Sect. 3.3.3).

\footnotetext{
2 From heasarc.gsfc.nasa.gov/FTP/fermi/data/lat/ weekly/spacecraft/
}

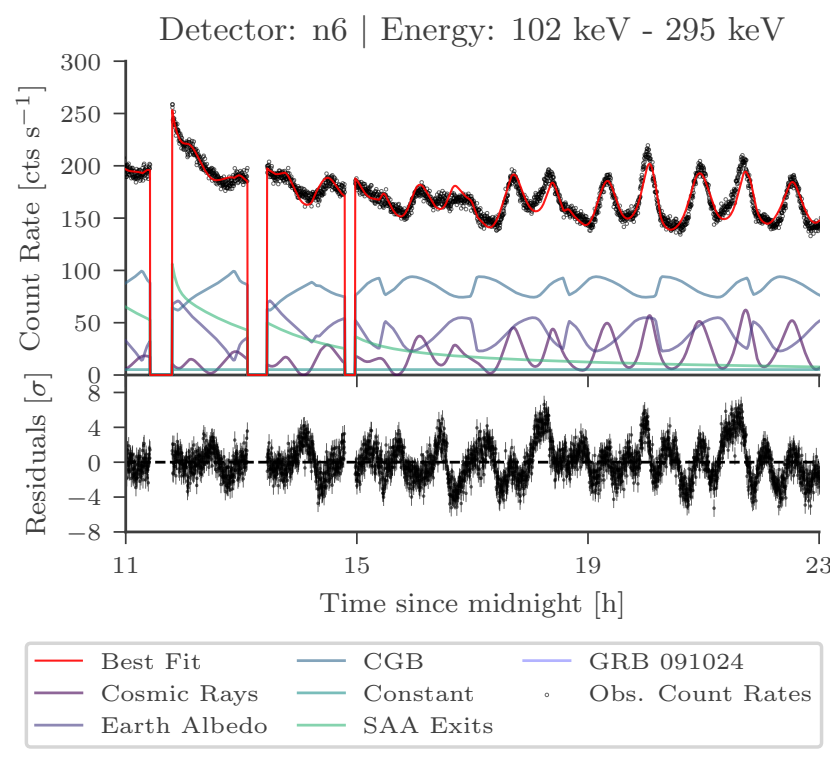

Fig. 11. Same as Fig. 10, but including the cosmic-ray component. For this plot the McIlwain $L$-parameter model is used.

Because the influence of cosmic rays on GBMs background is rising with energy, we can assume that in the high-energy channels of the BGO detectors the background is strongly dominated by cosmic rays. This can be used as an alternative to model the cosmic-ray contribution. To obtain the functional form, we binned the BGO data in the energy range of $8.6 \mathrm{MeV}$ to $16.6 \mathrm{MeV}$ to $100 \mathrm{~s}$ and fit a spline of third degree with a smoothing prior. It is then assumed that the variation in the amount of background rates caused by cosmic rays in the lower energies is linearly correlated with the functional obtained from the BGO data; therefore, we only fit for the normalization $C_{\mathrm{BGO} \text {-Approx }}$ that is multiplied with the fitted spline $S_{\mathrm{BGO}}$ as follows:

$R_{\mathrm{CR}}=C_{\mathrm{BGO}-\mathrm{Approx}} \cdot S_{\mathrm{BGO}}$.

The model obtained by the BGO approximation (Fig. 12) can fit the cosmic-ray contribution noticeably better than the model that uses the McIlwain L-parameter (Fig. 11). But of course we have to keep in mind that the BGO data is not free of other background sources because, for example, the spectrum of the Earth albedo (introduced in Sect. 3.3.3) extends to the $\mathrm{MeV}$ range. This shortfall could be overcome by the use of the anti-coincidence detector (ACD) of the LAT to model the cosmic-ray background in GBM. The ACD is primarily used to detected charged particles in 89 tiles around the LAT to exclude the charged particle induced background in the LAT data. Because the count rates of the ACD are also stored, we could use the different tiles to reconstruct the particle flux variation for different sites of the satellite individually. Unfortunately, the data from ACD is not publicly available, which is why we leave this approach for future improvements of the model and use the BGO approximation in the following sections.

The BGO approximation is the only empirical model component in this paper because of the complex processes that lead to the background contribution by cosmic rays, such as their deflection in the earth magnetic field and how they activate the satellite material. An appropriate procedure to deal with this would be a full simulation, but unfortunately there is no public mass model of the Fermi satellite that could be used to set up such a simulation. 


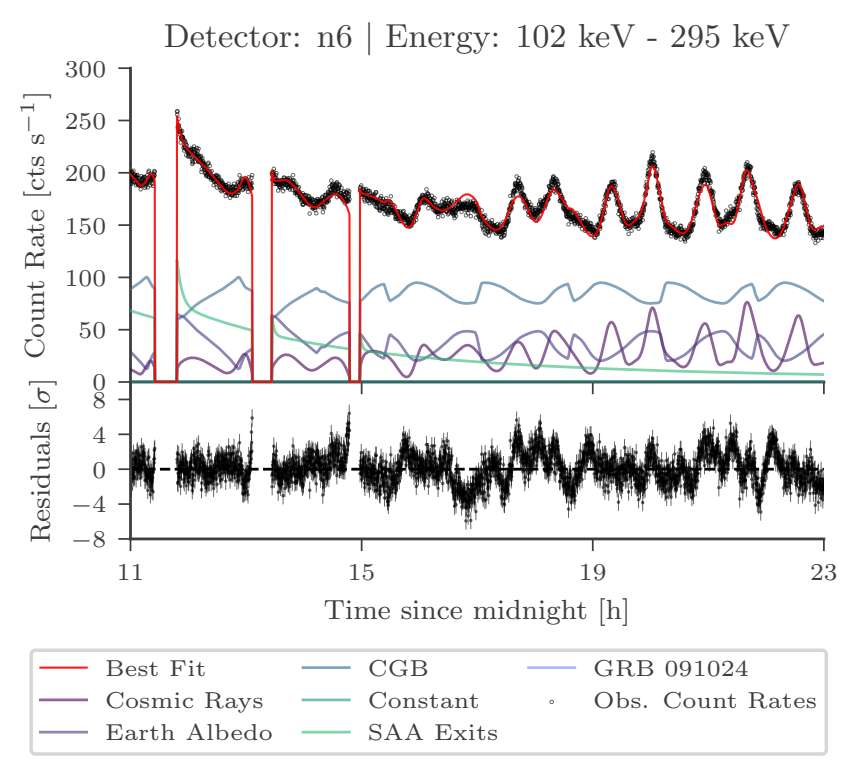

Fig. 12. Same as Fig. 10, but including the cosmic-ray component. For this plot the BGO approximation model is used.

\subsubsection{Point sources}

The CGB is not completely homogeneous because of a plethora of resolved point-like sources. Additionally there are very bright galactic point sources such as the Crab nebula. Their influence is more significant in the lower energy channels up to $100 \mathrm{keV}$ as most sources are thermal and have a spectrum rapidly falling with increasing energy. These point sources can be seen in the background of the detectors of GBM as shown in Fig. 13. To reduce the computational time we only take one point source into account, namely the Crab, but we plan to add more point sources in the future. The spectrum of the Crab as described in Madsen et al. (2017) is used (see Eq. (11)) and we again fit the normalization $C$ to the data as follows:

$\frac{\mathrm{d} N}{\mathrm{~d} E}=\frac{C}{E^{2.1}}$.

\subsubsection{Sun}

The Sun can be treated as a special point-like source, which also emits X-rays in the low keV range. The spectrum was investigated in Hannah et al. (2007), where $2 \sigma$ upper limits are given for the photon flux of the quiet Sun from $3 \mathrm{keV}$ to $200 \mathrm{keV}$. Even if these upper limits were the actual spectrum of the Sun, the influence of the Sun would be very small and, if at all, only visible in the lowest detector channels of GBM.

An exception are the eruptions of the Sun, when it violently releases a large amount of particles and photons with higher energies. While the particles of these so-called solar flares are deflected by the geomagnetic field, the photons reach GBM and are clearly visible in the data. Information about the flares can be obtained from the yearly flare files that are produced by the National Center for Environmental Information (NCEI). The solar flares can be modeled by an exponential decay function, which is set to zero up to the time when the flare arrives. To keep the number of parameters manageable we do not include solar flares in the model for now. If there is a strong solar flare we simply exclude this time interval from the analysis. The quiet Sun is modeled as a simple power-law spectrum in the following.

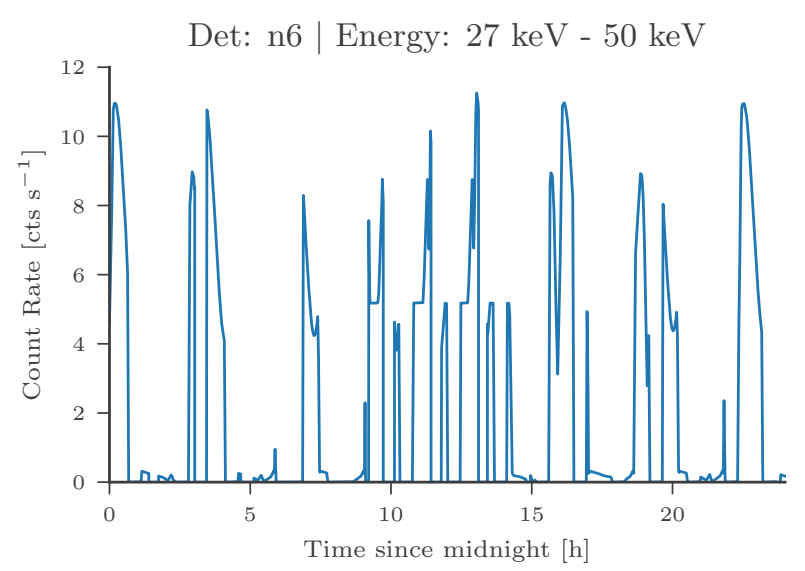

Fig. 13. Expected temporal count rate variation for the reconstructed energy range $27 \mathrm{keV}-50 \mathrm{keV}$ caused by the Crab Nebula with a spectrum as given in Eq. (11) with $C=9.7$ (Madsen et al. 2017).

\subsection{Fitting}

The resulting background model has a total of 50 free parameters for one day, one reconstructed energy channel and one detector, including the following:

- Two normalization and two decay constants for every SAA exit $(\approx 40$ parameters in total, depending on the number of SAA exits during the day).

- Two normalization and two decay constants for leftover excitation at the beginning of the day (4 parameters).

- Normalization for the Earth albedo, CGB, Sun, point sources (only Crab at the moment), and cosmic-ray contribution (5 parameters).

- One constant

We fitted the free parameters of our model to the data using the Bayesian nested sampler MultiNest (Feroz et al. 2009; Buchner et al. 2014).

As our model should represent the total count rates of GBM, we do not have a background signal. Thus, the correct likelihood is a Poisson likelihood without background, such as the following equation given in Cash (1979):

$\log (\mathcal{L})=\sum_{\text {time bins }}\left[N_{\text {model }}(t)-N_{\text {data }}(t) \cdot \log \left(N_{\text {model }}(t)\right)\right]$.

The parameter estimation of our model with the data of one day and one reconstructed energy channel takes $\approx 1: 30 \mathrm{~h}$ with 32 cores (Intel(R) Xeon(R) Platinum 8168 CPU @ 2.70 GHz) producing full posterior distributions. A Bayesian way of checking the ability of the model to describe the observed data are posterior predictive checks (PPC) (Gabry et al. 2019; Burgess et al. 2019). By the use of the posterior predictive distribution,

$\pi\left(y^{\mathrm{sim}} \mid y^{\mathrm{obs}}\right)=\int \pi\left(y^{\mathrm{sim}} \mid \theta\right) \pi\left(\theta \mid y^{\mathrm{obs}}\right) \mathrm{d} \theta$,

where $\pi\left(\theta \mid y^{\mathrm{obs}}\right)$ is the posterior distribution of $\theta$ and $\pi\left(y^{\operatorname{sim}} \mid \theta\right)$ the probability of a new simulated observation given a set of parameters $\theta$, we can generate simulated data and compare it to the original observed data. We sampled 300 parameter sets from the posterior distribution and simulated new data for each of these sets using the underlying Poisson distribution of photon counts. The observed data should be similar to the simulated data, which can visually be checked by making the area that contains $95 \%$ of the simulated data and verifying if the observed 
data lies within. There is strong indication that the fit is wrong if many observed data points lie outside of this marked area. This area that contains $95 \%$ of the simulated data is marked as green area around the best fit in the following.

The shown Poisson residuals are computed under the hypothesis that there is no uncertainty in the background. In other words, the probability of obtaining the observed counts is computed, given the expected counts from the background and then transforming it in units of sigma.

\section{Results}

To demonstrate the usefulness of our newly developed background model in a practical setting, we now provide several fits of the model to different days. In Sect. 4.1 we show how the model can be used to fit the background for the GRB 110920A for which the classical approach of using polynomial fits can give ambiguous answers. After this we show in Sect. 4.2 that the multiple emission peaks over more than $1000 \mathrm{~s}$ caused by the ultra-long GRB 091024 does not effect our fit even if we do not exclude the emission times from the fit. To check this for an even more extreme case, we show in Sect. 4.3 the background fit for the 21 June 2015, which was during the time of a V404 Cygni outburst. This outburst caused several very long emission periods seen in GBM during this day. We show that even such an extreme event only slightly disturbs our background fit and that we can identify more emission times than those that triggered the GBM. In the final example we show that we can identify untriggered excess emission for GRB 130925A with this background model.

\subsection{GRB 110920A}

GRB 110920A was a bright, single pulse GRB that occurred only about $100 \mathrm{~s}$ after an SAA exit of GBM. This causes the background to have a significant component from the exponential decay of the activated material. In Fig. 14 we show that our model can explain the background around GRB 110920A very well. For an accurate fit of the background during the source time of the GRB event we had to exclude the time of the GRB event from the fit, because it happened so shortly after an SAA exit that it disturbed the SAA exit fit badly. But we see the GRB as clear deviation from the background fits even without excluding the time, which means that this GRB could have been found with the background model without prior knowledge of the time of the GRB event.

In Fig. 15 we show the problems that can occur when the classical polynomial approach is used for a background that can not really be fitted by a polynomial because it contains a significant component from the exponential decay of the activated material after the SAA exit. The figure shows different polynomial fits, for different background time selections around the GRB event. The background fits are quiet different during the active time of the GRB, but all of the fits look equally valid in a short time around the GRB event $( \pm 50 \mathrm{~s})$. This random choice of one of these background polynomials can be overcome with our background model, which gives only one unambiguous answer.

\subsection{Ultra-long GRB 091024}

In this subsection we present the background fits for 24 October 2009, which contains the previously reported ultralong GRB 091024 (Gruber et al. 2011). GRB 091024 was

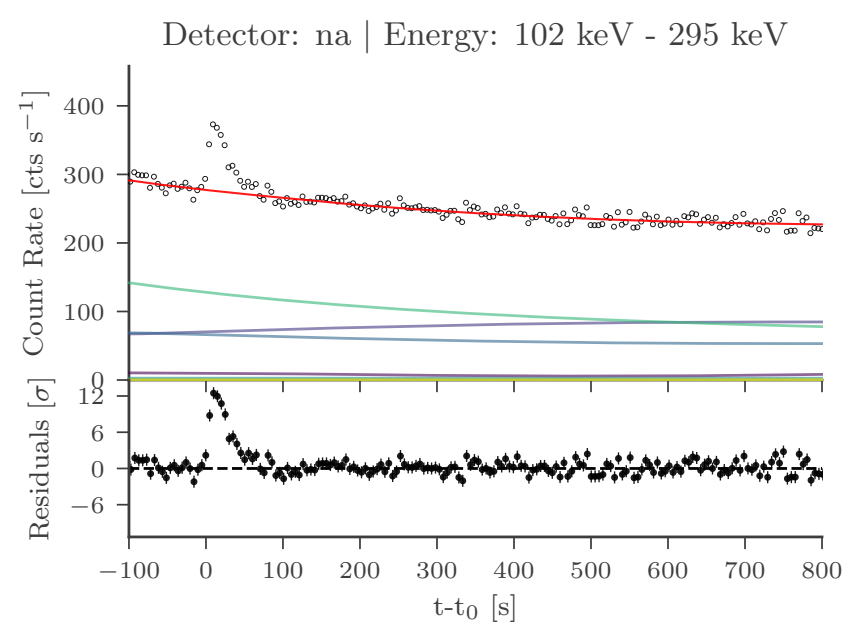

Fig. 14. Background fit for the time around GRB 110920A in the reconstructed energy range of $102-295 \mathrm{keV}$ for detector na with a time-bin size of $5 \mathrm{~s}$.

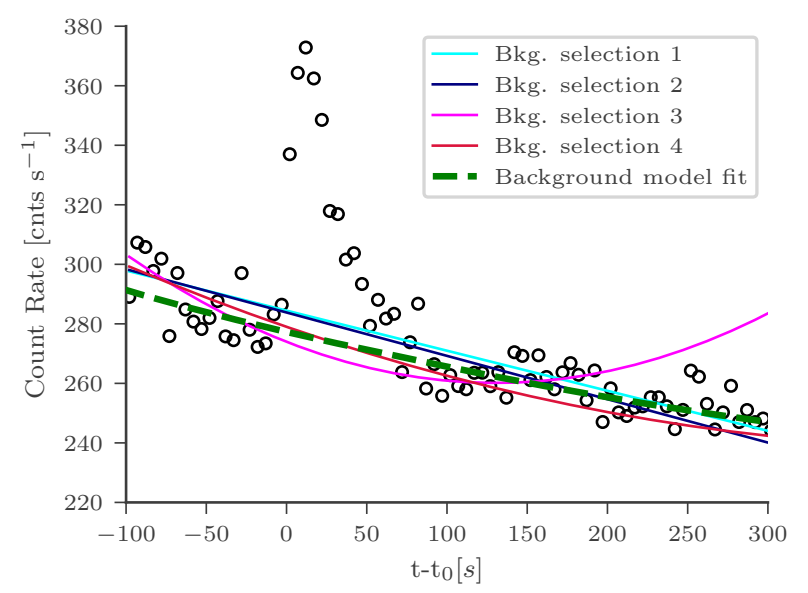

Fig. 15. Data and different background estimations for the time around GRB 110920A in the reconstructed energy range from 102 to $295 \mathrm{keV}$ for detector na with a time-bin size of $5 \mathrm{~s}$. The four solid lines are derived with the classical approach of fitting polynomials to the time before after the transient event, each with a different definition of these times $(1:-100 \mathrm{~s}$ to $-90 \mathrm{~s}$ and $150 \mathrm{~s}$ to $200 \mathrm{~s} ; 2:-100 \mathrm{~s}$ to $-70 \mathrm{~s}$ and $50 \mathrm{~s}$ to $200 \mathrm{~s} ; 3$ : $-100 \mathrm{~s}$ to $0 \mathrm{~s}$ and $100 \mathrm{~s}$ to $200 \mathrm{~s} ; 4:-100 \mathrm{~s}$ to $-50 \mathrm{~s}$ and $400 \mathrm{~s}$ to $500 \mathrm{~s}$ ). Additionally the best fit of our background model is plotted as a dashed green line.

detected by Fermi-GBM (Bissaldi \& Connaughton 2009), SwiftBAT (Marshall et al. 2009), and Konus-Wind (Golenetskii et al. 2009), consisting of multiple emission peaks with a total duration of about $1000 \mathrm{~s}$. The last emission peak had a duration of about $230 \mathrm{~s}$, which makes it an ideal test case to evaluate if this long, multiple emission period could have affected our background fit.

Figures 16 and 17 show the data and the best fit of the total background model for the two reconstructed energy ranges $27-50 \mathrm{keV}$ and $102-295 \mathrm{keV}$ for detector $\mathrm{n} 8$. The lower energy range is, as previously discussed, dominated by the CGB and the Earth albedo, while the cosmic-ray component is negligible. In the reconstructed energy range from 102 to $295 \mathrm{keV}$ the cosmicray component is already important.

It is clearly visible in the residuals and the posterior predictive check that the model can explain most of the observed data very well. In both reconstructed energy ranges an excess of count 


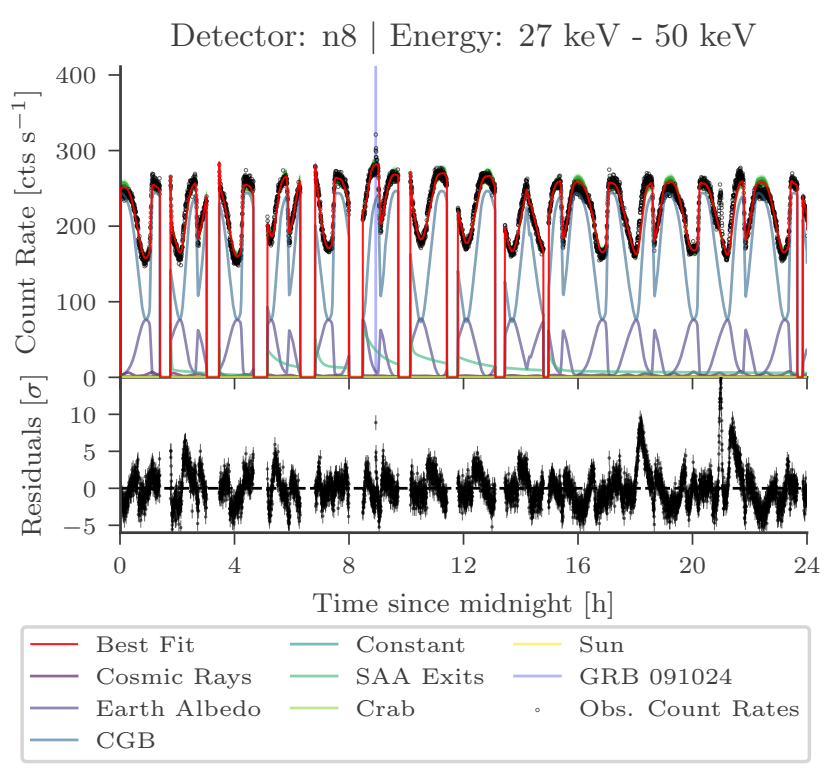

Fig. 16. Data and background fit for 24/10/2009 including the ultra-long GRB 091024 for detector $n 8$ in the reconstructed energy range of 27$50 \mathrm{keV}$ with a time-bin size of $15 \mathrm{~s}$. The plot shows the influence of the different sources in different colors, the best-fit total model in red, and the detected count rates in black. The GBM trigger time of GRB 091024 is indicated by a cyan line.

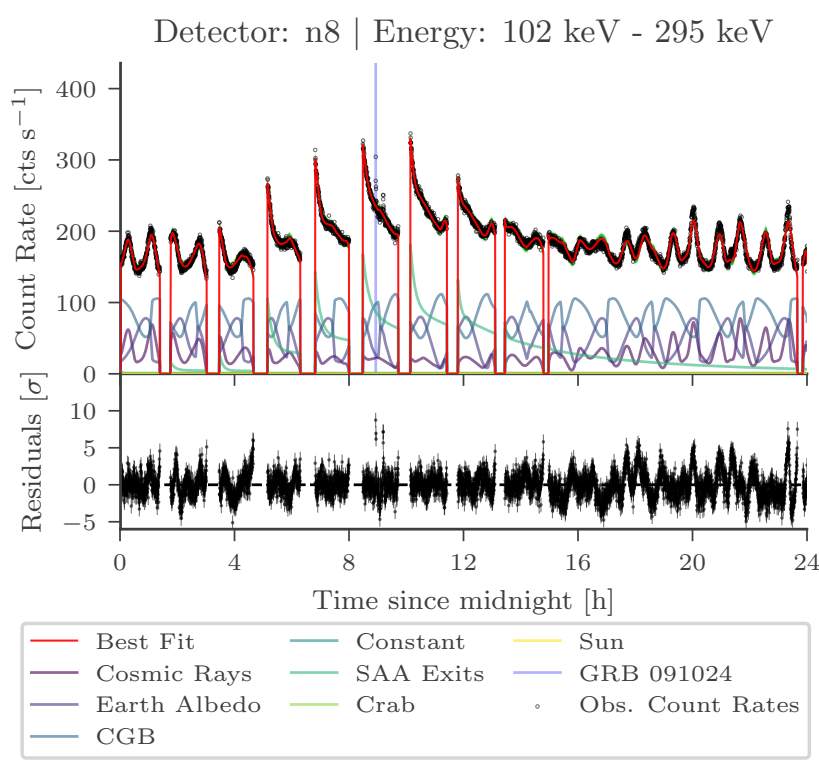

Fig. 17. Data and background fit for 24/10/2009 including the ultralong GRB 091024 for detector $n 8$ in the reconstructed energy range of $102-295 \mathrm{keV}$ with a time-bin size of $15 \mathrm{~s}$. The GBM trigger time of GRB 091024 is indicated by a cyan line.

rate for the trigger time is already visible in Figs. 16 and 17, but is much more obvious in Fig. 18, which shows the background fit for three different detectors and two different reconstructed energy ranges around the trigger time of GRB 091024. In this case, the multiple emission peaks with a total duration of more than $1000 \mathrm{~s}$ are clearly visible as deviations from the background fit, demonstrating the usefulness of a physically motivated background model to identify long-duration emission and the possibility of using the fitted background model as background estimation during the active time of the transient source. Owing to the long duration of GRB 091024, the detector with the smallest offset angle to the GRB position has changed from the first to the last peak from n8 to n0. This explains why the last peak is clearly visible in the light curves of n0 while the first peak is not.

We can also see a second strong deviation from the fitted background model in the low-energy range at about 21 hours after midnight, for which we checked that it is not from the same location as GRB 091024. This could be the result of a soft transient source.

Additionally, there are two long-duration, significant (up to 10 sigma) deviations from the fitted background model in the reconstructed energy range $27-50 \mathrm{keV}$. This could be due to a missing point source in our model, as we see this deviation in three detectors, which point in a similar direction (n6, n7 and n8) at similar (but not equal) times. To remove such deviations, we plan to include more known point sources in the background model in the future.

\subsection{V404 Cygni flaring}

In June 2015 V404 Cygni went into outburst, producing multiple flares which GBM triggered on (Jenke et al. 2016); 21 June was a very active day, on which GBM triggered ten times. We present the light curves of GBM for two energy channels and three detectors for part of the day in Fig. 19. We indicate the times of all GBM triggers with blue vertical lines. It is clearly shown from the PPCs and the residuals that on this day there were several more long and weak emission periods, which did not trigger GBM. We indicate some of these times roughly with red vertical lines in the plots. While we have not determined the sky positions of all these additional emission events, the similarity in the gamma-ray spectrum to that of the triggered events makes the V404 Cyg origin very likely. V404 Cyg was so active on that day that it disturbed the background fit a bit because the background fit is slightly too high for the non-active times of V404 Cygni. But still the several emission periods are very visible in the plots, even though we did not exclude the times when V404 Cygni was active from the fit. This is because we used physical derived background sources and not arbitrary polynomials and therefore we very strongly restricted which kind of shapes can be fit by our background model. This makes the presented background model a promising tool to search for long source emissions in the data of GBM in the future. If we wanted to use the fitted background model as an accurate background estimation during the active times of the source, we should of course redo the analysis and exclude the times when the source was active from the fit to get rid of this disturbance.

\subsection{Identifying untriggered excess emission}

Since our background model is fit over a full day, the fit parameters have no room to allow variations between individual satellite orbits, that is, to accommodate spatially (e.g., transient sources) or temporally (e.g., disturbances in the magnetic field) varying sources. This makes the background fit very stiff, and thus allows us to readily identify variable sources. This is demonstrated for September 25, 2013, when multiple emission periods were detected over several thousand seconds from the ultralong GRB 130925A (e.g., Golenetskii et al. 2013). In Fig. 20 the data for detector $\mathrm{n} 9$ and four reconstructed energy ranges are displayed, as well as our fitted model (red). The two GBM triggers are marked as vertical lines. The other emission period(s), 
B. Biltzinger et al.: A physical background model for the Fermi Gamma-ray Burst Monitor
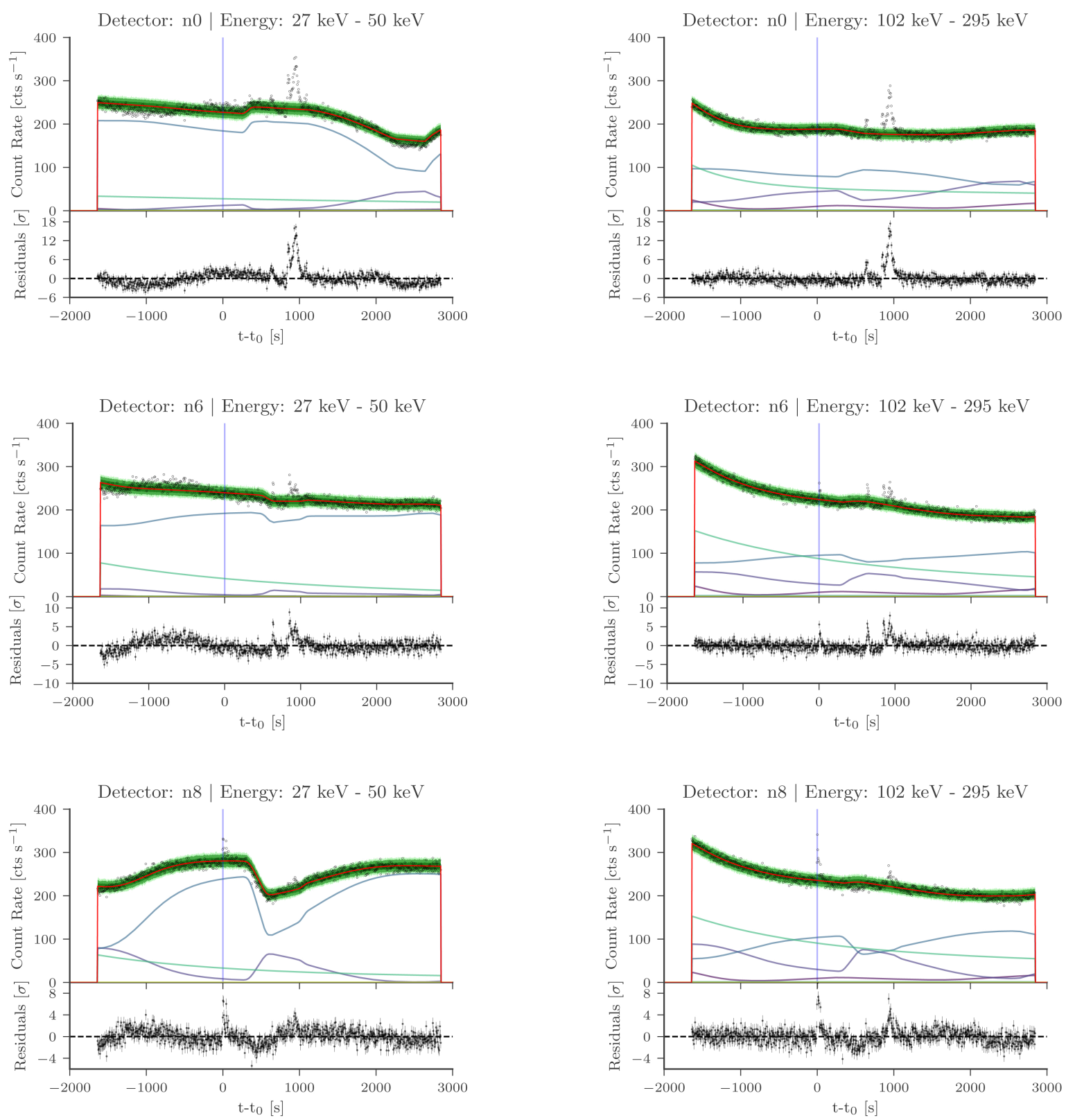

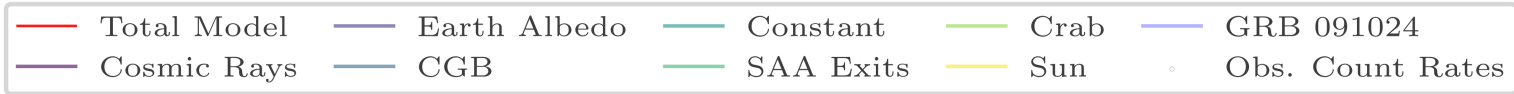

Fig. 18. Data and background fits for the $5000 \mathrm{~s}$ around the GBM trigger time of GRB $091024(t=0)$ for three different detectors: (n0 (top), n6 (middle), $\mathrm{n} 8$ (bottom)), and two reconstructed energy ranges (27-50 keV (left) and 102-295 keV (right)).

nicely covered by Konus-Wind, verify the identification of late GRB 130925A emission with GBM ( $\approx 5000$ s after the first GBM trigger).

\subsection{Comparison to Fermi GBM Orbital Background Subtraction Tool}

The advantage of the presented background model with respect to the Fermi GBM Orbital Background Subtraction Tool ${ }^{3}$ (OSV) (Fitzpatrick et al. 2012) is also demonstrated for 25 Septem-

\footnotetext{
3 https://fermi.gsfc.nasa.gov/ssc/data/analysis/user/ osv_1.3.tar
}

ber 2013 (Fig. 20), where the estimation of the OSV is overplotted in dark blue. Our model is capable of explaining the background variations in all four reconstructed energy ranges, whereas the OSV fails, mainly due to an autonomous repoint request (ARR) after the second GBM trigger. But also during days without ARR, the OSV method has its limitations (Figs. 21 and 22) because it depends on having no deviation of any kind (e.g. transients) also 30 orbits before and after the day in question. In Fig. 21 the OSV method fails because of the many strong and long emission periods that V404 Cygni produced 30 orbits before and after the shown time.

In Fig. 22 the OSV estimation fails during the first $10000 \mathrm{~s}$ as a consequence of an ARR, but gets better after the satellite 

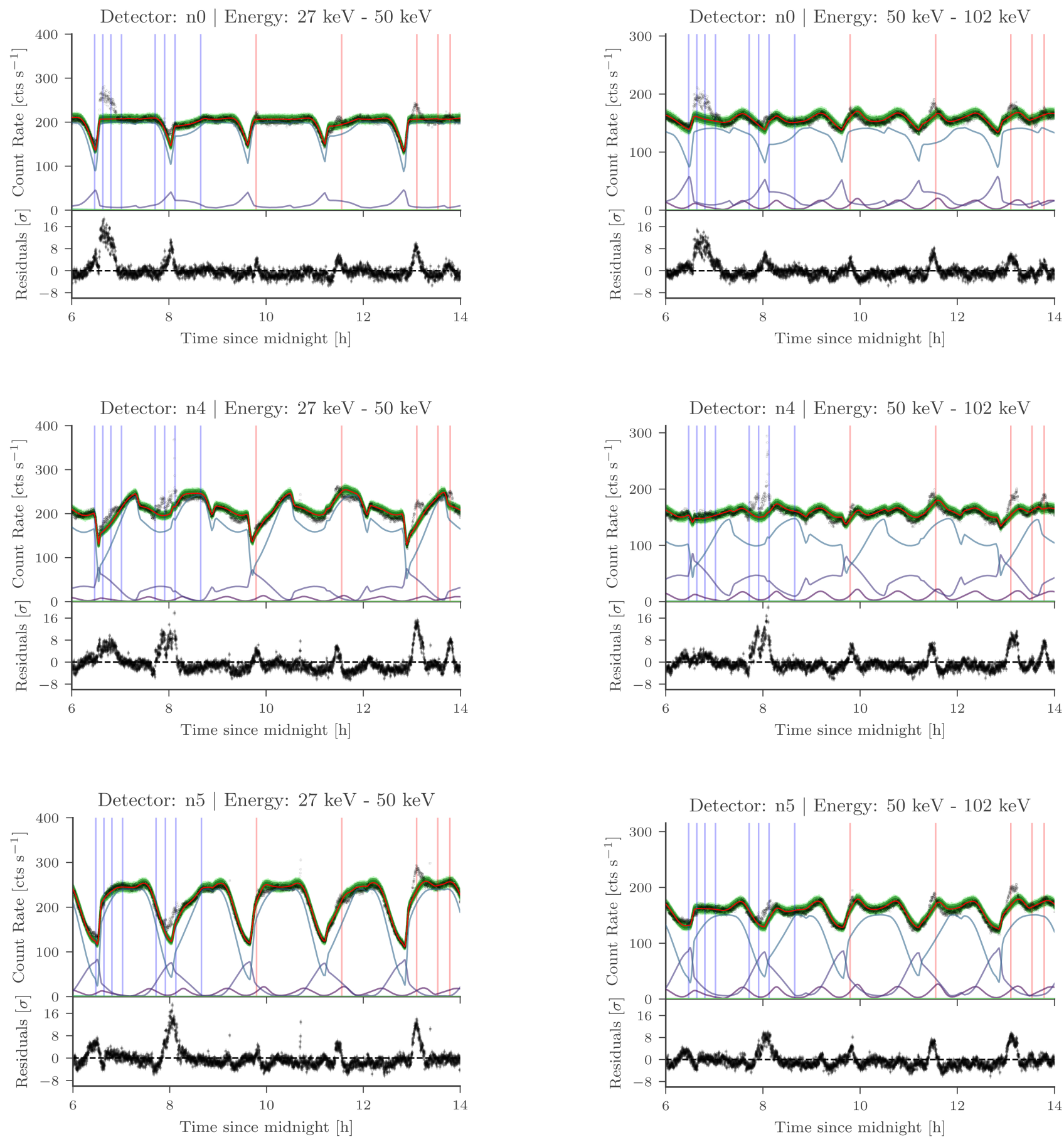

$\begin{array}{lllll}- \text { Total Model } & - \text { Earth Albedo } & - \text { Constant } & \text { Crab } & - \text { GRB } 091024 \\ \text { Cosmic Rays } & \text { CGB } & \text { SAA Exits } & \text { Sun } & \text { Obs. Count Rates }\end{array}$

Fig. 19. Data and background fits for part of the 21 June 2015, which was during a V404 Cygni outburst for 3 different detectors (n0 (top), n4 (middle), n5 (bottom)) and 2 reconstructed energy ranges $(27-50 \mathrm{keV}($ left $)$ and $50-102 \mathrm{keV}($ right $))$. The blue vertical lines indicate GBM trigger times, whereas the red vertical lines indicate possible new emission events that did not trigger GBM.

returns to its normal pointing mode. But it does not get better than our model, which can fit all the data well. For about 1000 s between $\approx 6000$ and $\approx 7000$ s the OSV cannot estimate the background at all owing to a SAA passage 30 orbits before or after this time. The high excess of data above the background fit at $\approx 7000 \mathrm{~s}$ is well visible in the energy channels up to $100 \mathrm{keV}$ in several detectors and will be investigated in the future.

With about 1 onboard trigger every 10 orbits $^{4}$, this limitation affects the background estimation for some period of time on $\sim 30 \%$ of all days.

4 https://gammaray.nsstc.nasa.gov/gbm/ 


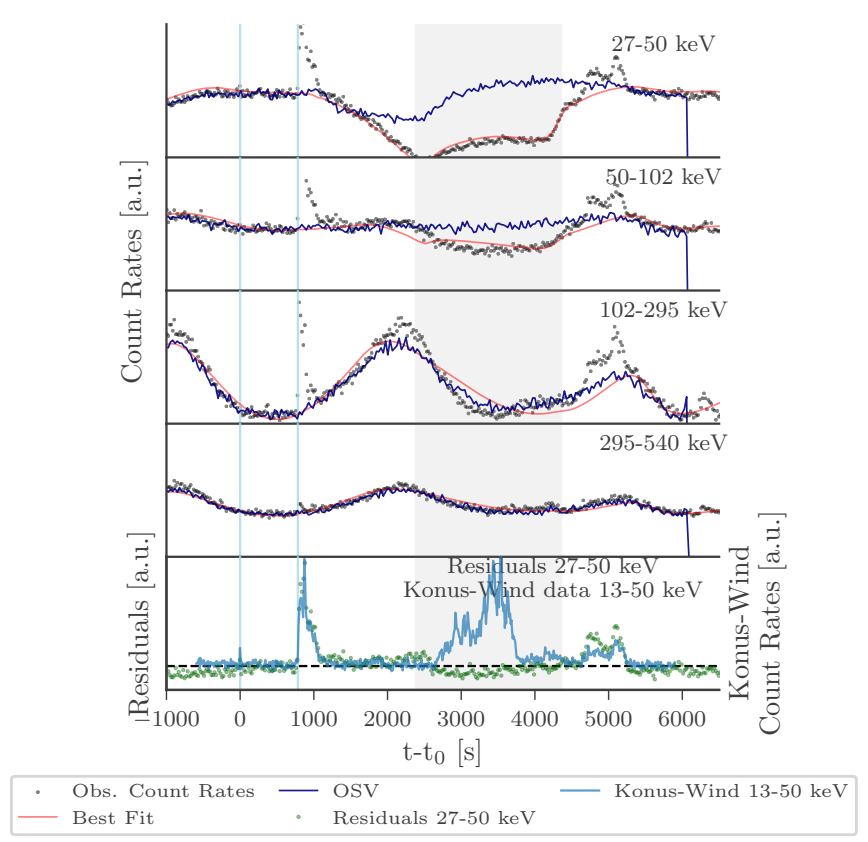

Fig. 20. Data (black), background fit (red), and background estimation (OSV, dark blue) with the OSV (Fitzpatrick et al. 2012) for the emission time of $130925 \mathrm{~A}$, detector $\mathrm{n} 9$ and 4 reconstructed energy ranges in the top 4 panels. In the bottom panel the residuls for the background fit between $27 \mathrm{keV}$ and $50 \mathrm{keV}$ reconstructed energy are given, as well as the background subtracted Konus-Wind data between $13 \mathrm{keV}$ and $50 \mathrm{keV}$ (Golenetskii et al. 2013). The two blue vertical lines show the time of the two GBM triggers for GRB 130925A and the gray area the time during which the position of GRB 130925A was occulted by the earth for GBM.

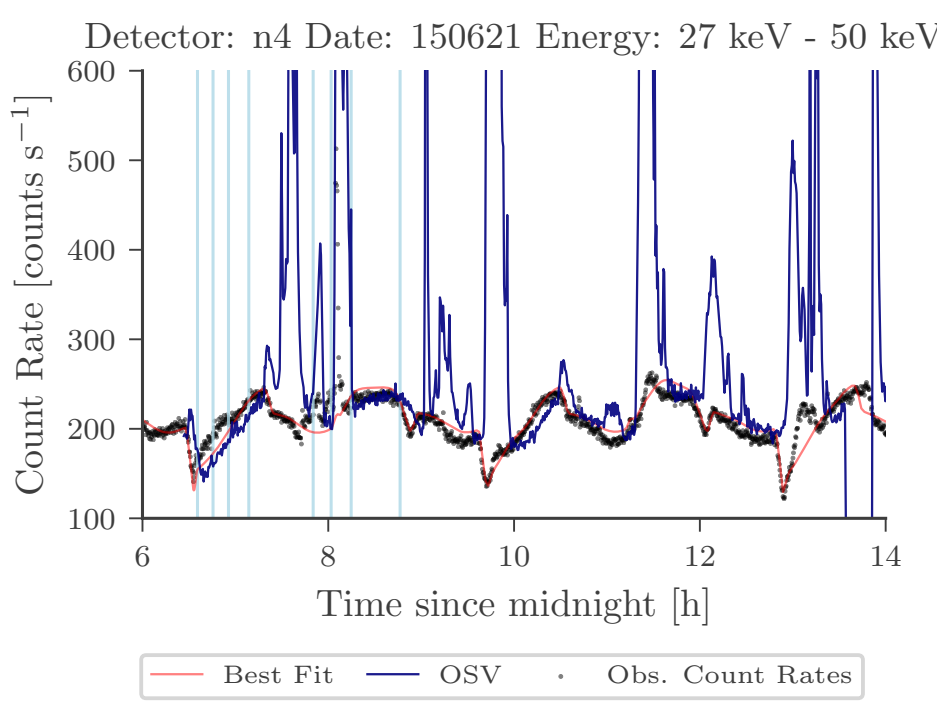

Fig. 21. Data (black), background fit (red), and background estimation (OSV, dark blue) with the OSV for part of 21 June 2015 during the 2015 V404 Cygni outburst, detector $n 9$ and reconstructed energy between $27 \mathrm{keV}$ and $50 \mathrm{keV}$. All GBM triggers during this time are shown as blue vertical lines (before the onboard triggers were turned off).

The following summarizes the advantages of our physical model. The model (1) is not affected by ARR or other deviations from the normal pointing mode; (2) is not affected if the satellite was in a SAA 30 orbits before or after the time of interest; (3) is not affected by GRBs or any transient sources occurring 30

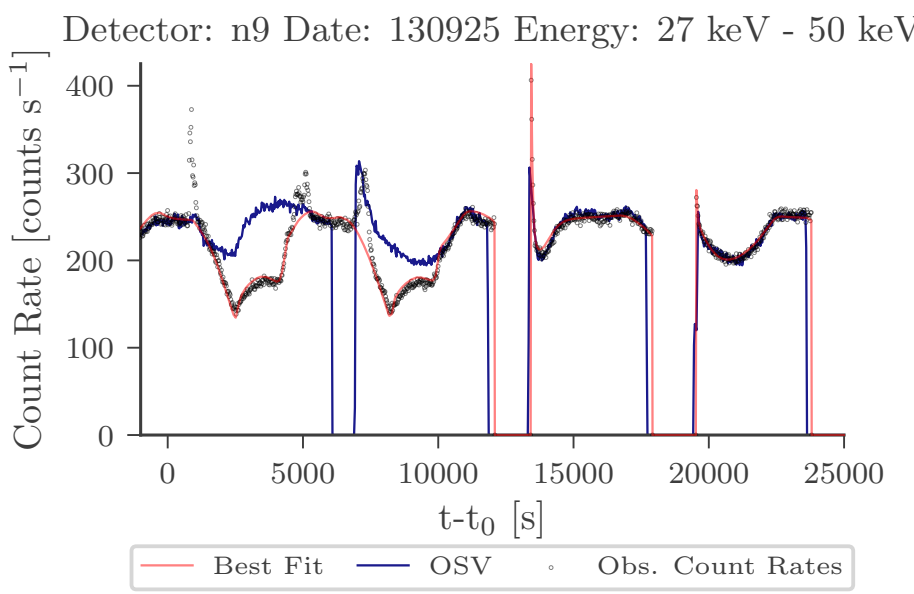

Fig. 22. Data (black), background fit (red), and background estimation (OSV, dark blue) with the OSV for $25000 \mathrm{~s}$ on the 25 September 2013 , the detector $\mathrm{n} 9$ and reconstructed energy are between $27 \mathrm{keV}$ and $50 \mathrm{keV}$.

orbits before or after the time of interest; (4) is a more robust method that for example does not need the radiation environment or magnetic field to be stable for at least three days, owing to the \pm 30 orbits that are used in the OSV; (5) treats the data in a statistically correct way, which allows us to derive proper errors on the background counts; and (6) allows us to understand and study the different components of the background, which is also of interest for future missions.

\section{Conclusions}

The derivation of a physically motivated background model for GBM was presented. It consists of six different source components (the Earth albedo, CGB, Sun, point sources (just the Crab at the moment), SAA, and cosmic rays), where only the cosmicray contribution is modeled empirically.

The presented model is capable of modeling the background for most of the GBM PHA channels as shown for the example cases of the ultra-long GRB 091024, the V404 Cygni outburst in June 2015 and GRB 110920A.

There are three obvious possible future applications of this physically motivated background model: (1) Use it as a new approach to search for ultra-long GRBs in the more than 12 years of GBM data, which until now have been indistinguishable from the background variations; (2) Infer information about the different background components, for example the CGB spectrum in the GBM energy range; and (3) Develop a transient search algorithm for slowly rising or long transients that are not recognized by the onboard trigger algorithm.

Acknowledgements. J. Michael Burgess acknowledges support by the Alexander von Humboldt foundation.

\section{References}

Abdo, A. A., Ackermann, M., Ajello, M., et al. 2009, Phys. Rev. D, 80, 122004

Ajello, M., Greiner, J., Sato, G., et al. 2008, ApJ, 689, 666

Berlato, F., Greiner, J., \& Burgess, J. M. 2019, ApJ, 873, 60

Bissaldi, E., \& Connaughton, V. 2009, GCN Circ., 10070

Bissaldi, E., von Kienlin, A., Lichti, G., et al. 2009, Exp. Astron., 24, 47

Bouchet, L., Strong, A. W., Porter, T. A., et al. 2011, ApJ, 739, 29

Buchner, J., Georgakakis, A., Nandra, K., et al. 2014, A\&A, 564, A125 
Burgess, J. M. 2019, GBM DRM Generator

Burgess, J. M., Yu, H.-F., Greiner, J., \& Mortlock, D. J. 2018, MNRAS, 476, 1427

Burgess, J. M., Greiner, J., Bégué, D., \& Berlato, F. 2019, MNRAS, 490, 927 Cash, W. 1979, ApJ, 228, 939

Churazov, E., Sazonov, S., Sunyaev, R., \& Revnivtsev, M. 2008, MNRAS, 385

da Silva, M. R., \& Rocco, E. M. 2017, J. Phys.: Conf. Ser., 911, 012005

Diehl, R., Siegert, T., Greiner, J., et al. 2018, A\&A, 611, A12

Feroz, F., Hobson, M. P., \& Bridges, M. 2009, MNRAS, 398, 1601

Fitzpatrick, G., McBreen, S., Connaughton, V., Briggs, M., \& Team, t. G., 2012, Proc, SPIE, 84433B

Gabry, J., Simpson, D., Vehtari, A., Betancourt, M., \& Gelman, A. 2019, J. R. Stat. Soc. A, 182, 389

Ganushkina, N. Y., Dandouras, I., Shprits, Y. Y., \& Cao, J. 2011, J. Geophys. Res. (Space Phys.), 116, A09234

Giacconi, R., Gursky, H., Paolini, F. R., \& Rossi, B. B. 1962, Phys. Rev. Lett., 9, 439

Golenetskii, S., Aptekar, R., Frederiks, D., et al. 2013, GCN Circ., 15260

Golenetskii, S., Aptekar, R., Mazets, E., Pal'shin, V., \& Frederiks, D. 2009, GCN Circ., 10083

Greiner, J., Burgess, J. M., Savchenko, V., \& Yu, H.-F. 2016, ApJ, 827, L38

Gruber, D., Krühler, T., Foley, S., et al. 2011, A\&A, 528, A15

Hannah, I. G., Hurford, G. J., Hudson, H. S., Lin, R. P., \& van Bibber, K. 2007, ApJ, 659, L77

Hasinger, G. 2004, Nucl. Phys. B Proc. Suppl., 132, 86
Hoover, A. S., Kippen, R. M., Meegan, C. A., et al. 2005, Nuovo Cimento C Geophys. Space Phys. C, 28, 797

Horiuchi, S., Ng, K. C. Y., Gaskins, J. M., Smith, M., \& Preece, R. 2015, ArXiv e-prints [arXiv:1502.03399]

Jenke, P. A., Wilson-Hodge, C. A., Homan, J., et al. 2016, ApJ, 826, 37

Kippen, R., Hoover, A. S., Wallace, M., et al. 2007, AIP Conf., Proc, 921

Madsen, K. K., Forster, K., Grefenstette, B. W., Harrison, F. A., \& Stern, D. 2017, ApJ, 841, 56

Marshall, F., Baumgartner, W., Beardmore, A., et al. 2009, GCN Circ., 10062

McIlwain, C. E. 1966, in Radiation Trapped in the Earth's Magnetic Field, ed. B. M. McCormac (Dordrecht: Springer, Netherlands)

Meegan, C., Lichti, G., Bhat, P. N., et al. 2009, ApJ, 702, 791

Mozzoni, D. T. 2007, PhD Thesis, The Florida State University

Pendleton, G. N., Briggs, M. S., Kippen, R. M., et al. 1999, ApJ, 512, 362

Pesce-Rollins, M. 2009, ArXiv e-prints [arXiv:0912 . 3611]

Petry, D. 2005, AIP Conf. Proc., 745, 709

Schaefer, R. K., Paxton, L. J., Selby, C., et al. 2016, Space Weather, 14, 330

Shea, M. A., Smart, D. F., \& Gentile, L. C. 1987, Phys. Earth Planet. Inter., 48, 200

Szécsi, D., Bagoly, Z., Kóbori, J., Horváth, I., \& Balázs, L. G. 2013, A\&A, 557, A8

Thompson, D. J., Simpson, G. A., \& Ozel, M. E. 1981, J. Geophys. Res., 86, 1265

van Allen, J. A., \& Frank, L. A. 1959, Nature, 183, 430

Worsley, M. A., Fabian, A. C., Bauer, F. E., et al. 2005, MNRAS, 357, 1281 